\title{
Sobrecarga, afrontamiento y salud en cuidadoras de pacientes con demencia tipo Alzheimer
}

\section{Chronic stress, coping and health in caregivers of Alzheimer's disease patients}

\author{
Gilberto Aldana \\ Leticia Guarino ${ }^{1}$ \\ Hospital José María Vargas, Venezuela Universidad Simón Bolívar, Venezuela
}

(Rec: marzo de 2012 - Acep: mayo de 2012)

\begin{abstract}
Resumen
El presente estudio relacionó las variables sobrecarga del cuidador y los estilos de afrontamiento con la salud general percibida en 300 cuidadoras de personas con demencia tipo Alzheimer en estadio II, divididas en 220 cuidadoras informales y 80 cuidadoras formales no profesionales, evaluadas en la sede nacional de la Fundación Alzheimer de Venezuela, ubicada en la ciudad de Caracas - Venezuela. Entre los resultados más relevantes destaca que las cuidadoras informales presentan mayores niveles de sobrecarga, en comparación con las cuidadoras formales no profesionales. Esto se asoció a un mayor compromiso en la salud percibida de aquellas, que específicamente presentaron mayores síntomas somáticos y de ansiedad. En cuanto a los estilos de afrontamiento, se encontró que las cuidadoras informales usan significativamente más afrontamiento emocional y menos afrontamiento racional y por desapego, que las cuidadoras formales no profesionales, lo que lleva a comprometer más la salud de las primeras. Finalmente, los indicadores de salud están directamente relacionados con mayor sobrecarga, de tal forma que a mayor sobrecarga, mayor deterioro, lo que se observa con mayor magnitud en las cuidadoras informales.
\end{abstract}

Palabras clave: demencia tipo Alzheimer, cuidadoras informales, cuidadoras formales no profesionales, sobrecarga, afrontamiento y salud general.

\begin{abstract}
The present study related the caregivers chronic stress and the coping styles with the perceived general health of 300 caregivers of type II Alzheimer's patients, divided in 220 informal caregivers and 80 non professionals formal caregivers, evaluated at the National centre of the Venezuelan Alzheimer Fundation, in Caracas-Venezuela. Among the most relevant results it was found that the informal caregivers presented higher levels of chronic stress, compared with the non professionals formal caregivers, and this was related with a deterioration of the health status of formers, who specifically presented higher symptons of somatization and anxiety. Regarding the coping styles, it was found that the informal caregivers use significally more emotional coping and less rational and detacthment coping than teh non professionals formal caregivers. Finally, the health indexes are directly related with higer chronic stress, so higher stress is related with higher deterioration and this is particularly true in informal caregivers.

Keywords: Alzheimer dementia, informal caregivers, non professional formal caregivers, chronic stress, coping and general health.
\end{abstract}

\footnotetext{
1 Correspondencia dirigida a Leticia R. Guarino. Universidad Simón Bolívar, Dpto. de Ciencia y Tecnología del Comportamiento. Edificio de Estudios Generales, 1er. Piso. Valle de Sartenejas, Aptdo. Postal 89.000 - A. Edo. Miranda. Venezuela. Tel.: 0058 212 9063595. E-mail: lguarino@usb.ve.
} 


\section{Introducción}

Se estima que hoy en día en el mundo entero existen 24,3 millones de personas con demencia y cada año se suman aproximadamente 4,6 millones de nuevos casos. Los cálculos establecen que se presenta un nuevo caso de demencia cada siete segundos y que para el año 2040 el mundo tendrá 81,1 millones de personas con demencia, de los cuales entre el 50 y 55\% serán debido a la demencia senil tipo Alzheimer-DTA (Alzheimer's Disease International, 2009).

En América Latina se calcula que en la actualidad 1,8 millones de personas padecen de demencia y para el año 2040 se alcanzarían los casos de demencia reportados actualmente en la América del Norte, es decir, 9 millones de personas, de los cuales aproximadamente de 4,5 a 5 millones de casos son correspondientes a la demencia tipo Alzheimer (Alzheimer's Disease International, 2009). Por otra parte, la Fundación Alzheimer de Venezuela (2009) reportó que en nuestro país aproximadamente 140.000 venezolanos sufren de algún tipo de demencia, de los cuales 75.000 se deben a la demencia tipo Alzheimer (DTA).

La psicología de la salud en el abordaje de la DTA, al igual que con otras enfermedades crónicas, considera la evaluación, estudio y tratamiento, tanto del paciente como de la persona que ejerce su cuidado, ya que ambas se convierten en víctimas de este trastorno (Aldana-Sierralta, 2003; Aldana-Sierralta, 2009 y Brannon y Feist, 2001). En toda enfermedad crónica, como es el caso de la DTA, suele existir una persona (familiar o profesional) que asume el papel de cuidador principal, quien debe "dedicar gran parte de su tiempo y esfuerzo para permitir que la persona cuidada pueda desenvolverse en la vida diaria" (Marco, 2004, p. 44).

El cuidar a una persona con DTA implica una gran variedad de actividades y responsabilidades altamente demandantes y estresantes, inclusive en mayor escala que en los casos de cuidado de pacientes con retardo en el desarrollo, pacientes con cáncer, con accidentes cerebro-vasculares y con depresión (Garrido y Tamai, 2006).

Entre las alteraciones fisiológicas que pueden sufrir los cuidadores familiares de enfermos de Alzheimer tenemos: disminución del funcionamiento del sistema inmunológico (Kielcot-Glaser, Glaser, Shuttleworth, Dyer, Ogrocki y Speicher, 1987), alteraciones cardiovasculares (King, Oka y Young, 1994, c.p. Laserna, Castillo, Peláez, Vavío, Torres, Rueda, Ramírez y Pérez, 1997), trastornos gastrointestinales, trastornos osteomusculares y dolores asociados (Gallart y Connell,
1998, c.p. Rodríguez, 2005) o cambios en algunas variables metabólicas (Vitaliano, Scanlan, Krenz, Schwartz y Marcovina, 1996, c.p. Laserna et al, 1997).

Con relación a las alteraciones psicológicas, se ha encontrado que los cuidadores familiares sufren distintos grados de depresión, ansiedad, conflictos familiares (Conde-Sala 1998; Laserna et al, 1997; Rodríguez, 2005), pérdida de su autoestima y disminución de su autoeficacia (Laserna et al 1997; Rodríguez, 2005), y finalmente agresividad hacia el paciente $\mathrm{u}$ otras personas (Conde - Sala, 1998; Laserna et al, 1997; MarmaneuMoliner, 2007; Rodríguez del Álamo, 2002).

Para entender la situación del cuidado del paciente con DTA y su impacto sobre el cuidador, se debe considerar que ésta es una patología neurológica crónica y que la supervivencia dura aproximadamente entre 8 y 14 años, siendo hasta la fecha incurable además de degenerativa, por lo que la dependencia que adquiere el paciente de su cuidador va aumentando, al igual que la dedicación que debe cumplir este último (Pearlin, Mullan, Semple y Skaff, 1990). Desde el punto de vista del modelo bio-psico-social, se considera que esta situación deteriora cada vez más la salud del cuidador, pues él mismo debe enfrentarse a una gran cantidad de estresores, los cuales se relacionan con la "sobrecarga", término con el que se conoce al fenómeno del estrés en el ámbito del cuidado (Pearlin, 1994; Zarit, 1987; 1996).

El modelo de estrés aplicado al cuidado, propuesto por Pearlin et al (1990), engloba de manera esquemática las relaciones entre los antecedentes del contexto del cuidador (características socioeconómicas, historia y relaciones del cuidador), los estresores primarios (deterioro cognitivo, funcional y conductual del paciente), así como la sobrecarga y deprivación relacional del cuidador frente al paciente, los estresores secundarios (tensión psicológica y por ejercicio del rol), los mediadores (el afrontamiento y apoyo social) y finalmente el impacto del estrés sobre la salud del cuidador. Para completar este modelo, Hooker, Frazier y Monahan (1994) sugirieron la inclusión de la personalidad dentro de los factores mediadores.

Un concepto clave en el modelo de Pearlin et al (1990), es el denominado contención de estrés, proceso por el cual los cuidadores limitan o disminuyen los efectos de los estresores primarios y secundarios mediante la utilización de variables mediadoras, siendo éstos los recursos psicológicos que disponen los cuidadores para interactuar con esos estresores, una de ellos el afrontamiento, el cual fue una variable considerada en la presente investigación. 
Específicamente en lo que respecta al afrontamiento, éste ha sido una variable que ha recibido moderada atención por parte de los investigadores en su papel mediador del estrés en el cuidador (Aramburu, Izquierdo y Romo, 2001; Cooper, Katona, Orrel y Livingstone, 2006; Goode, Haley, Roth y Ford,1998; Haley, Roth, Coleton, Ford, Best, Collins y Isobe, 1996; Irigoyen, Goñi y Biurrun, 2002; Montorio, Yanguas y Díaz, 1999; Neundorfer, 1991; Pearlin y Schooler, 1978). Desde una perspectiva disposicional, los estilos de afrontamiento se refieren a predisposiciones personales para hacer frente a las situaciones y son los responsables de las preferencias individuales en el uso de uno u otro tipo de estrategia de afrontamiento, así como de su estabilidad temporal y situacional (Fernández-Abascal, 1997). En lo que respecta al cuidador, la presencia de un determinado estilo de afrontamiento debería estar relacionado con niveles de salud diferenciales, tomando en consideración el carácter moderador de la relación salud-enfermedad que tiene esta variable. Para la presente investigación se trabajará con el modelo de afrontamiento descrito por Roger, Jarvis y Najarian (1993), quienes clasifican a los estilos de afrontamiento por desapego emocional y racional como estilos adaptativos, mientras que a la evitación y al afrontamiento emocional como estilos desadaptativos.

Asimismo, otra variable importante que se consideró en este estudio fue el tipo de cuidador, a saber, informal y/o formal. Generalmente, el cuidador informal de una persona con demencia es un familiar que asume la mayor responsabilidad del cuidado, con poca capacitación para el mismo, no recibe remuneración económica, ofrece atención sin limites de horarios y tiene un elevado grado de compromiso (Garrido y Tamai, 2006; Marco, 2004; Velásquez 2006). No obstante, también se encuentra la figura del cuidador formal, quien por su preparación se dedica al cuidado de la persona con Alzheimer como parte de su trabajo en un horario establecido y recibe honorarios por las tareas que realiza (Marco, 2004). Estas características generalmente se toman como base para considerar que los cuidadores familiares o informales soportan una mayor sobrecarga y por lo tanto un mayor compromiso en su salud y bienestar en comparación con los cuidadores profesionales o formales. Cabe destacar que en esta investigación se usó la clasificación ampliada por Fernández-Puebla (2007), quienes hablan de cuidadores informales y cuidadores formales no profesionales, refiriéndose a estos últimos como aquellos cuidadores pagados, que no tienen una formación académica para el rol que desempeñan y laboran en un horario determinado. Esta clasificación surge como consecuencia de la existencia de personas dedicadas al cuidado como forma de empleo remunerado, pero que no cuentan con ningún adiestramiento para el ejercicio de este rol.

Estas consideraciones llevaron a plantearnos como objetivo principal de esta investigación determinar la relación entre la sobrecarga, los estilos de afrontamiento, la condición del cuidador (formal e informal) y la salud percibida de cuidadoras de pacientes con DTA en la población venezolana.

\section{Método}

\section{Participantes}

La muestra que conformó este estudio fue de 300 participantes divididos en 220 cuidadoras informales y 80 cuidadoras formales no profesionales. Todas las cuidadoras evaluadas asistieron a la sede nacional de la Fundación Alzheimer de Venezuela y entre los criterios de inclusión de la muestra tenemos los siguientes:

a- Sexo: femenino (mayoritariamente son mujeres quienes cumplen el rol de cuidador tanto informal como formal no profesional).

b- Edad: de 30 años en adelante.

c- Ausencia de transtornos psiquiátricos y/o consumo de psicofármacos.

d- Ubicación del paciente cuidado en un estadio II de la DTA.

e- Puntuación del paciente en el Examen Mínimo del Estado Mental (MMS) entre 13 y 20 puntos.

Las edades de las participantes se distribuyeron de la siguiente forma: 120 con edades comprendidas entre 30 y 50 años, 145 con edades entre 51 y 70 años y 35 con más de 70 años.

\section{Instrumentos}

- $\quad$ Escala de Sobrecarga del Cuidador de Zarit (1980), adaptado al castellano por Martín, et al, (1996). Esta escala evalúa la sobrecarga o estrés de los cuidadores de pacientes con demencia desde el punto de vista de la teoría transaccional del estrés. Consta originalmente de 22 ítems que evalúan las repercusiones negativas que conlleva el cuidado sobre la vida de quien ejerce esta tarea. Para esta investigación, se realizó una adaptación de la Escala de Zarit. Luego de este proceso, la escala quedó constituida por dos sub-dimensiones: 
a- Sobrecarga Subjetiva: integrada por los siguientes 10 ítems: 1-2-3-4-6-10-11-12-13-17.

$b$ - Sobrecarga Objetiva: conformada por los siguientes 6 ítems: 7-15-16-19-20-21.

Los índices de consistencia interna (Alfa de Cronbach) son de un $\alpha=0,94$ para la sub-escala subjetiva y un $\alpha=0,85$ para la sub-escala objetiva. En la medida que la persona obtenga un puntaje más alto, se asume que reporta mayores niveles de sobrecarga. La escala permite también obtener un puntaje total.

- Cuestionario CSQ (Coping Styles Questionarie, de Roger, Jarvis y Najarian, 1993), traducido y adaptado a la población venezolana por Guarino, Sojo y Bethelmy (2007): Este cuestionario consta de 40 ítems y mide las siguientes dimensiones de afrontamiento al estrés: Emocional (12 ítems), Evitación (5 ítems), Desapego Emocional (13 ítems) y Racional (10 ítems).

La validación de este instrumento fue llevada a cabo en una muestra venezolana por Guarino, Sojo y Bethelmy (2007). Entre los resultados tenemos que la consistencia interna (Alfa de Cronbach) de cada una de las dimensiones fue: Emocional: $(\alpha=0.78)$, Evitación: ( $\alpha=0.65)$, Desapego Emocional: $(\alpha=0.73)$ y Racional: $(\alpha=0.78)$. La forma de responder es mediante una escala Likert de 4 puntos donde $0=\operatorname{Nunca}(\mathrm{N}), 1=$ Algunas veces (A), $2=$ Frecuentemente (F) $3=$ Siempre (S). La puntuación más alta dentro de las categorías denotan el estilo de afrontamiento más predominante en el participante.

- Cuestionario de Salud General (Goldberg, 1978) (GHQ28), adaptado al castellano por Lobo, Pérez-Echeverría y Artal (1986): Este cuestionario fue diseñado para ser utilizado como un test de screaning autoadministrado, destinado a detectar trastornos psíquicos entre los encuestados en un ámbito comunitario y en medios clínicos no psiquiátricos (Goldberg, 1978; Iraurgi, 2002). Su propósito es detectar aquellas formas de trastorno psicológico que pudieran tener relevancia en la práctica médica y por tanto se centra en los componentes psicológicos de una mala salud (Iraurgi, 2002).

El GHQ-28 se deriva del análisis factorial del GHQ-60 y se compone de 4 subescalas: síntomas somáticos, ansiedad e insomnio, disfunción social y depresión grave. A través de numerosos estudios de validez con entrevistas clínicas, se concluye una sensibilidad del $84 \%$ y una especificidad del $82 \%$. En cuanto al punto de corte aconsejado, en 16 estudios de validez el rango abarca de un valor mínimo de 4 ó 5 , a un máximo de 11 ó 12, siendo el más frecuentemente utilizado el de 4 ó 5 (Iraurgi, 2002). En el caso de la presente investigación, se utilizó una versión de 28 ítems previamente empleada con población venezolana y los resultados se interpretan de tal forma que menores puntajes indican mayor presencia del síntoma.

\section{Procedimiento}

El investigador se puso en contacto con cada uno de los cuidadores informales y formales no profesionales de pacientes con demencia tipo Alzheimer que asisten a la Unidad de Psicología de la Salud de la Fundación Alzheimer de Venezuela. Tras explicarles los objetivos de este estudio, se les solicitó a las cuidadoras interesadas que firmaran un consentimiento que certificara su participación voluntaria en la investigación. Luego de que el cuidador (familiar o no familiar, dependiendo del caso) firmó el consentimiento, se procedió a la aplicación de los instrumentos de medida correspondientes, a saber: la Escala de Sobrecarga del Cuidador de Zarit (adaptado a la población venezolana), el Cuestionario de Estilos de Afrontamiento (CSQ) y el Cuestionario de Salud General de Goldberg (1978) (GHQ28). Esta actividad se realizó en la Unidad de Psicología de la Salud de la Fundación Alzheimer, habiendo sido los propios autores de esta investigación quienes aplicaron los cuestionarios de manera individualizada a las participantes.

\section{Resultados}

La tabla 1 muestra los valores descriptivos de la variable sobrecarga, para cada uno de los grupos de cuidadoras.

Se puede evidenciar que el grupo de las cuidadoras informales presenta mayor sobrecarga en comparación con el grupo de las cuidadoras formales no profesionales. Este resultado fue estadísticamente significativo para cada tipo sobrecarga: subjetiva (U Mann-Whitney $=3710,000 ; \mathrm{p}<0.0001)$, objetiva (U Mann-Whitney $=4694,000 ; \mathrm{p}<0.0001$ ) y total (U MannWhitney=3791,500; $<<0.0001)$.

En lo que respecta a los estilos de afrontamiento, la tabla 2 presenta los resultados descriptivos para cada uno de los estilos evaluados, a saber: racional, emocional, desapego y evitación.

En el grupo de cuidadoras informales, el estilo de afrontamiento con la media más alta es el "racional", seguido de "evitación", "emocional" y finalmente el "desapego" . Por su parte, el grupo de las cuidadoras 
formales no profesionales muestra una tendencia ligeramente diferente a la anterior. El estilo de afrontamiento más empleado es el "racional", seguido de "evitación", luego "desapego" y finalmente "emocional". Todas estas diferencias resultaron estadísticamente significativas, tal como se muestra en la tabla 3, lo cual sugiere que las cuidadoras informales usaron, en general, estilos de afrontamiento menos funcionales como el emocional, mientras usaron en menor proporción el estilo racional y por desapego, con respecto a las cuidadoras formales no profesionales.

En relación a la variable salud, evaluada a través del GHQ, los resultados descriptivos para cada grupo se muestran en las tablas 4 y 5 , respectivamente.

El síntoma con mayor presencia en las cuidadoras informales es la ansiedad, seguido de somatización y

Tabla 1.

Descriptivos para la sobrecarga en ambas cuidadoras.

\begin{tabular}{ccccccc}
\hline & \multicolumn{3}{c}{ Cuidadoras Informales } & \multicolumn{3}{c}{$\begin{array}{c}\text { Cuidadoras Formales } \\
\text { No Profesionales }\end{array}$} \\
\cline { 2 - 7 } & $\begin{array}{c}\text { Sobrecarga } \\
\text { Subjetiva }\end{array}$ & $\begin{array}{c}\text { Sobrecarga } \\
\text { Objetiva }\end{array}$ & $\begin{array}{c}\text { Sobrecarga } \\
\text { Total }\end{array}$ & $\begin{array}{c}\text { Sobrecarga } \\
\text { Subjetiva }\end{array}$ & $\begin{array}{c}\text { Sobrecarga } \\
\text { Objetiva }\end{array}$ & $\begin{array}{c}\text { Sobrecarga } \\
\text { Total }\end{array}$ \\
\hline Media & 3,03 & 3,25 & 3,14 & 2,14 & 2,59 & 2,36 \\
Desv. Típ. & 0,85 & 0,75 & 0,7 & 0,82 & 0,76 & 0,7 \\
Mínimo Posible & 1 & 1 & 1 & 1 & 1 & 1 \\
Máximo Posible & 5 & 5 & 5 & 5 & 5 & 5 \\
$\mathrm{~N}$ & 220 & 220 & 220 & 80 & 80 & 80 \\
\hline
\end{tabular}

Tabla 2.

Descriptivos para los estilos de afrontamiento en ambas cuidadoras.

\begin{tabular}{ccccccccc}
\hline & \multicolumn{3}{c}{ Cuidadoras Informales } & \multicolumn{4}{c}{ Cuidadoras Formales No Profesionales } \\
\hline & Racional & Emocional & Evitación & Desapego & Racional & Emocional & Evitación & Desapego \\
\hline Media & 2,60 & 2,22 & 2,25 & 1,91 & 2,88 & 1,95 & 2,62 & 2,33 \\
Desv. Típ. & 0,55 & 0,51 & 0,61 & 0,41 & 0,55 & 0,52 & 0,76 & 0,43 \\
Mínimo Posible & 1 & 1 & 1 & 1 & 1 & 1 & 1 & 1 \\
Máximo Posible & 4 & 4 & 4 & 4 & 4 & 4 & 4 & 4 \\
$N$ & 220 & 220 & 220 & 220 & 80 & 80 & 80 & 80 \\
\hline
\end{tabular}

Tabla 3.

Prueba U de Mann Whitney para el contraste de las puntuaciones en los estilos de afrontamiento según el tipo de cuidadora.

\begin{tabular}{lcccc}
\hline & Racional & Emocional & Evitación & Desapego emocional \\
\hline U de Mann-Whitney & 6306,000 & 5994,500 & 6229,500 & 3890,500 \\
W de Wilcoxon & 30616,000 & 9234,500 & 30539,500 & 28200,500 \\
Z & $-3,759$ & $-4,231$ & $-3,885$ & $-7,404$ \\
Sig. Asintót. (bilateral) & .000 & .000 & .000 & .000 \\
\hline
\end{tabular}


Tabla 4.

Resultados para las dimensiones de salud del GHQ para el grupo de cuidadoras informales.

\begin{tabular}{lccccc}
\hline & Somatización & Ansiedad & Disfunción Social & Depresión & Salud General \\
\hline Media & 2,68 & 2,52 & 2,75 & 3,61 & 2,89 \\
Desv. Típ. & 0,72 & 0,68 & 0,47 & 0,49 & 0,47 \\
Mínimo Posible & 1,00 & 1,00 & 1,00 & 1,00 & 1,00 \\
Máximo Posible & 4,00 & 4,00 & 4,00 & 4,00 & 4,00 \\
N & 220 & 220 & 220 & 220 & 220 \\
\hline
\end{tabular}

disfunción social. El síntoma que menos presencia tiene dentro de este grupo es el de depresión. Finalmente, en lo que respecta a la percepción de la salud general, el grupo la percibe moderadamente comprometida. Recordemos que esta escala tiene la particularidad que los puntajes se analizan de manera descendente, es decir, a mayor puntación obtenida es menor la presencia del síntoma y por lo tanto mayor es el nivel de salud percibida.

En el grupo de cuidadoras formales no profesionales, la somatización es el síntoma con mayor presencia, seguido de disfunción social y ansiedad, mientras que la depresión se muestra como el síntoma menos reportado. Finalmente, en lo que respecta a la salud general, este grupo reporta unos mejores niveles de salud, en comparación con el grupo de las cuidadoras informales.

Al comparar los resultados de ambos grupos de cuidadoras, se obtienen diferencias significativas para cada síntoma, así como para el puntaje total, lo que indica que las cuidadoras informales mostraron un mayor deterioro en su salud física y mental, comparadas con las cuidadoras formales no profesionales.

Finalmente, las tablas 7 y 8 , respectivamente, muestran los niveles de correlación entre la sobrecarga en cada una de sus dimensiones y los factores o indicadores de salud del cuestionario GHQ de Goldberg, para cada grupo de cuidadoras.

Tabla 5.

Resultados para las dimensiones de salud del GHQ para el grupo de cuidadoras informales.

\begin{tabular}{lccccc}
\hline & Somatización & Ansiedad & Disfunción Social & Depresión & Salud General \\
\hline Media & 3,05 & 3,18 & 3,10 & 3,79 & 3,28 \\
Desv. Típ. & 0,69 & 0,73 & 0,44 & 0,35 & 0,46 \\
Mínimo Posible & 1,00 & 1,00 & 1,00 & 1,00 & 1,00 \\
Máximo Posible & 4,00 & 4,00 & 4,00 & 4,00 & 4,00 \\
$\mathrm{~N}$ & 80 & 80 & 80 & 80 & 80 \\
\hline
\end{tabular}

Tabla 6.

Prueba U de Mann Whitney para el contraste de las puntuaciones Salud General, según el tipo de cuidador.

\begin{tabular}{lccccc}
\hline & Somatización & Ansiedad & Depresión & Disfunción Social & Salud General \\
\hline U de Mann-Whitney & 6160,000 & 4313,000 & 6557,000 & 4605,500 & 4381,500 \\
W de Wilcoxon & 9400,000 & 7553,000 & 9797,000 & 7845,500 & 7621,500 \\
Z & $-3,982$ & $-6,767$ & $-3,486$ & $-6,361$ & $-6,651$ \\
Sig. Asintót. (bilateral) & .000 & .000 & .000 & .000 & .000 \\
\hline
\end{tabular}


Estos resultados sugieren que las cuidadoras informales con mayores puntajes en sobrecarga (en cualquiera de sus dimensiones), reportan mayor somatización, ansiedad, depresión, disfunción social y en general, un mayor deterioro en su estado de salud.

Estos puntajes resultaron significativos desde el punto de vista estadístico al nivel de 0,01 , a excepción de la asociación entre sobrecarga objetiva y ansiedad $(\mathrm{Rxy}=-.441)$, sobrecarga objetiva y depresión $(\mathrm{Rxy}=$ -.238) y sobrecarga subjetiva y disfunción social $(\mathrm{Rxy}=-.362)$, las cuales resultaron de menor magnitud. Finalmente, cuando relacionamos la sobrecarga objetiva con la disfunción social, la misma resultó no ser significativa desde el punto de vista estadístico. En general, si bien se hallan algunas asociaciones significativas entre las dimensiones de la sobrecarga y los indicadores de salud, éstas son de menor magnitud, comparadas con los observados para el grupo de cuidadoras informales.

\section{Discusión}

El cuidar a una persona con Alzheimer implica el cumplimiento de diversas actividades demandantes, debido a que estos pacientes presentan dificultades para el correcto seguimiento de órdenes y a su vez comienzan a manifestar problemas de comportamiento asociados a un mal manejo de la ansiedad o la depresión, evidenciándose esta problemática ya sea a través de trastornos conductuales, o por el procesamiento erróneo de la información que emana del ambiente (Pearlin et al, 1990).

El cuidar a una persona con Alzheimer genera altos niveles de compromisos bio-psico-sociales que pueden afectar la salud de quien ejerce este rol (Conde-Sala 1998; Laserna et al, 1997; Kiecolt-Glaser et al., 1987; Marmaneu-Moliner, 2007; Rodríguez, 2005; Rodríguez del Álamo, 2002). El cuidar no sólo implica la inversión de tiempo y energía para el cumplimiento de las

Tabla 7.

Correlación entre los factores de Sobrecarga y Salud General en Cuidadoras Informales.

\begin{tabular}{|c|c|c|c|c|c|}
\hline & Salud General & Somatización & Ansiedad & Disfunción Social & Depresión \\
\hline Sobrecarga Objetiva &,$- 415 * *$ &,$- 331 * *$ &,$- 412 * *$ &,$- 323 * *$ &,$- 267 * *$ \\
\hline Sobrecarga Subjetiva &,$- 621 * *$ &,$- 590 * *$ &,$- 592 * *$ &,$- 362 * *$ &,$- 391 * *$ \\
\hline Sobrecarga Total &,$- 594 * *$ &,$- 537 * *$ &,$- 571 * *$ &,$- 381 * *$ &,$- 356^{* *}$ \\
\hline
\end{tabular}

$\mathrm{N}=220$.

** La correlación es significativa al nivel 0,01 (bilateral).

Tabla 8.

Correlación entre los factores de Sobrecarga y Salud General en Cuidadoras Formales No Profesionales.

\begin{tabular}{|c|c|c|c|c|c|}
\hline & Salud General & Somatización & Ansiedad & $\begin{array}{c}\text { Disfunción } \\
\text { Social }\end{array}$ & Depresión \\
\hline Sobrecarga Objetiva &,$- 404 * *$ &,$- 304 * *$ &,$- 441^{*}$ &,- 159 &,$- 238 *$ \\
\hline Sobrecarga Subjetiva &,$- 723 * *$ &,$- 684 * *$ &,$- 673 * *$ &,$- 257 *$ &,$- 451 * *$ \\
\hline Sobrecarga Total &,$- 611 * *$ &,$- 532 * *$ &,$- 613 * *$ &,$- 223 * *$ &,$- 377 * *$ \\
\hline
\end{tabular}

$\mathrm{N}=80$.

** La correlación es significativa al nivel 0,01 (bilateral).

* La correlación es significativa al nivel 0,05 (bilateral). 
actividades derivadas del mismo, sino que también la persona que ejerce este rol debe ocuparse de las actividades pertinentes a su propia vida (Aldana-Sierralta, 2003).

En el presente estudio se encontró que las cuidadoras informales presentaron significativamente mayor nivel de sobrecarga, en comparación con las cuidadoras formales no profesionales. Asimismo, se comprobó la importancia de estudiar la sobrecarga por dimensiones, objetiva - subjetiva - total, y no sólo la sobrecarga general como propone Zarit (1980; citado por Martin et al., 1996), ya que esto permite discriminar los niveles de estrés primario de las cuidadoras en función de su percepción de la realidad.

En este sentido, ambos grupos de cuidadoras reportaron unos niveles elevados de sobrecarga objetiva, siendo el grupo de cuidadoras informales las que mayor incidencia de la misma presentaron. Este tipo de sobrecarga es concebida como las actividades concretas que desempeña la cuidadora (p.e., asear al paciente todos los días, darle de comer, etc) (García-Calvente et al, 2004; Pearlin, 1994; Zarit, 1996).

El puntaje de la sobrecarga subjetiva fue menor en el grupo total de cuidadoras evaluadas, sin embargo fue el grupo de las cuidadoras informales el que presentó significativamente un mayor puntaje en esta subdimensión. Recordemos que la sobrecarga subjetiva se relaciona con la forma en que se percibe la situación y en concreto, la respuesta emocional del cuidador ante la experiencia de ejercer ese rol, siendo definido como el sentimiento psicológico que se asocia al hecho de cuidar (García-Calvente et al., 2004; Pearlin, 1994; Zarit, 1996).

En síntesis, fue la sobrecarga objetiva la dimensión que más incidió en la elevación total de los niveles de sobrecarga general reportada por las cuidadoras formales no profesionales. No obstante, en las cuidadoras informales fueron ambas dimensiones, tanto la subjetiva como la objetiva, las que incrementaron el nivel de sobrecarga general y por lo tanto fue el grupo que comparativamente obtuvo mayores niveles de sobrecarga total.

Por su parte, el afrontamiento, considerado como uno de los más importantes mediadores en el proceso de estrés del cuidador (Deví y Almazán, 2002; Artaso, Goñi y Biurrun, 2002; Muela et al., 2002; Pearlin et al., 1990), también mostró diferencias en función del tipo de cuidadoras. En este sentido, las cuidadoras informales usaron significativamente menos el afrontamiento racional y desapego emocional, considerados estilos adaptativos, mientras que usaron más afrontamiento de tipo emocional, comparadas con las cuidadoras formales no profesionales. Este patrón es considerado perjudicial y desadaptativo, puesto que se asocia a un esquema de respuesta neurótica, catalogado en otros estudios como un factor de riesgo para la salud en general (Roger, 1995; Santed, Sandin, Chorot, Olmedo y García-Campayo, 2003; Guarino, 2009), lo cual en este estudio podría explicar el mayor compromiso en la salud de las cuidadoras informales.

Acerca de los indicadores de salud percibida, los resultados mostraron cómo las cuidadoras informales reportaron un mayor deterioro general, reflejado en puntajes significativamente mayores en ansiedad, depresión, somatización y disfunción social, a diferencia de las cuidadoras formales no profesionales, concluyéndose con esto que las primeras padecen más trastornos de salud en su rol de cuidadoras que las segundas, tal como se esperaba de acuerdo al modelo de sobrecarga y desgaste descrito por Pearlin et al (1990). Esto sugiere que el cuidado de un paciente con demencia tipo Alzheimer genera más deterioro y desgaste físico, psicológico y emocional a personas que están vinculadas afectivamente con el paciente y que no tienen ninguna formación previa en el ejercicio del rol del cuidador.

Finalmente y en relación a los niveles de asociación entre la sobrecarga y los indicadores de salud en ambos grupos de cuidadoras, en este estudio se encontraron correlaciones significativas entre las dos variables (sobrecarga y salud percibida) en los dos grupos, aunque llama la atención que para las cuidadoras informales las correlaciones son de mayor magnitud que para las cuidadoras formales no profesionales. Estos resultados confirman la existencia de una estrecha relación entre la sobrecarga y el deterioro en el estado de salud de las cuidadoras, tal y como lo han expuesto otras investigaciones (Devi y Almazan, 2002; Lozada, Montorio, Izal y Márquez, 2006; Roig, Abengózar y Serra, 1998). Sin embargo, sugiere que el deterioro es mayor en las cuidadoras informales al ejercer su rol, con respecto a las cuidadoras formales no profesionales.

En síntesis, esta investigación comprobó que existen diferencias significativas en cuanto a la percepción de la sobrecarga en el rol de cuidador, así como en el uso de formas de afrontamiento y en los indicadores de salud, entre cuidadoras informales y cuidadoras formales no profesionales, siendo las primeras las más afectadas en su equilibrio psicosocial con respecto a las segundas. Si bien ambos tipos de cuidadoras muestran más deterioro mientras mayor sea la sobrecarga, se hace evidente que el mayor peso en la labor del cuidado de 
pacientes con Alzheimer lo tienen las cuidadoras que, teniendo una vinculación afectiva con el paciente, no tienen una formación previa o entrenamiento en las tareas propias del rol (cuidadoras informales). Todo esto sugiere que las instituciones de salud gubernamentales deberían ofrecer mayores recursos para la formación de personal que se dedique al cuidado de pacientes con demencia tipo Alzheimer, formando a nivel profesional a las cuidadoras y dándoles estrategias de cuidado a los familiares que por circunstancias del entorno deben asumir tal responsabilidad.

\section{Referencias}

Aldana-Sierralta, G. (2003). El Psicólogo clínico en el abordaje de la demencia tipo Alzheimer: Evaluación e Intervención Conductual. Manuscrito no publicado, Universidad Central de Venezuela, Caracas.

Aldana-Sierralta, G. (2009, octubre). No hay tiempo que perder para cuidar a quien cuida un familiar con Alzheimer. Boletín de la Fundación Alzheimer de Venezuela, Capítulo Sucre, 1, (6), p.3-4.

Alzheimer`s Disease International (2009). Informe Mundial sobre el Alzheimer 2009. Resumen Ejecutivo de Londres.

Aramburu, I., Izquierdo, A. y Romo, I. (2001). Análisis comparativo de necesidades psicosociales de cuidadores informales de personas afectadas de Alzheimer y ancianos con patología no invalidante. Revista Multidisciplinaria de Gerontología, 11 (2), 64-71.

Artaso, B., Goñi, A. y Biurrun, A. (2002). Estrategias de Afrontamiento de las cuidadoras informales del paciente con demencia. Cuadernos de Medicina Psicosomática y Psiquiatría de Enlace, 60, 38-45.

Brannon L. y Feist J. (2000). Psicología de la Salud. Madrid: Paraninfo Thomson Learning.

Conde-Sala, J. (1998). Integración de la familia en el proceso terapéutico de la demencia. En Alberca, R y López, S. (eds.). Enfermedad de Alzheimer y otras demencias. Madrid: Panamericana.

Cooper, C., Katona, C., Orrell, M. y Livingstone, G. (2006). The role of coping strategies in predicting anxiety in caregivers of people with Alzheimers disease: the LASER-AD study. Journal of Affective Disorders, 9, 15-20.

Devi, J. y Almazan, R. (2002). Modelos de estrés y afrontamiento en el cuidador del enfermo con demencia. Revista Multidisciplinaria de Gerontología, 12 (1), 31-37.

Fernández-Abascal (1997). Estudio sobre la Escala de Estilos y Estrategias de Afrontamiento. Anales de Psicología, 12, 185-203

Fundación Alzheimer de Venezuela (2009, enero). Ayudando al que Ayuda. Boletín de la Fundación Alzheimer de Venezuela, $25,16-19$.

García-Calvente, M., Mateo, I y Maroto, G. (2004). El impacto de cuidar en la salud y la calidad de vida de las mujeres. Gaceta Sanitaria, 18, 83-92.

Garrido, R. y Tamai, S. (2006). Impacto Da Demencia Nos Cuidadores e Familiares: Relevancia Clinica E Escala de Avaliacao. En Bottino, C., Laks, J. y Blay, S. (eds.). Demencia e Trastornos Cognitivos en Idosos. Guanaba Hoogan: Río de Janeiro.

Goode, K., Haley, W., Roth D. \& Ford, G. (1998). Predicting longitudinal changes in caregiver physical and mental health: a stress process model. Health Psychology, 17, 190-198.
Guarino, L. (2009). Validación del Modelo de Sensibilidad Emocional. Propuesta de un Modelo de Mediación Moderada. Manuscrito no publicado. Universidad Simón Bolivar, Caracas, Venezuela.

Guarino, L., Sojo, V. y Bethelmy, L. (2007). Adaptacion y validación preliminar de la versión hispana del CSQ (Coping Style Questionnaire). Psicología Conductual, 15 (2), 173-189.

Haley, W., Roth, D., Coleton, M., Ford, G., West, C., Collins, R. \& Isobe, T. (1996). Appraisal, coping and social support as mediators of well being in black and white family caregivers of patients with Alzheimer`s disease. Journal of Consulting and Clinical Psychology, 64, 121-129.

Hooker, K., Fraizer, L.D. \& Monahan, D.J. (1994). Personality and coping among caregivers of spouses with dementia. The Gerontologist, 34 (4), 386-392.

Kielcot-Glaser, J., Glaser, R., Shuttleworth, E., Dyer C., Ogrocki, P. \& Speicher, C. (1987). Chronic stress ad immunity in family caregivers of Alzheimer's disease victims. Psychosomatic Medicine, 49, 523-535.

Laserna, Castillo, Peláez, Vavío, Torres, Rueda, Ramírez y Pérez (1997). Alteraciones Emocionales y Variables Moduladoras en Familiares-Cuidadores de Enfermos de Alzheimer. Psicología Conductual, 5 (3), 364-375.

Lobo, A., Pérez-Echeverría M. y Artal, J. (1986). Validity of the scaled version of the General Health Questionaire (GHQ-28) in a spanish population. Psychological Medicine, 16, 135-140.

Losada, A., Montorio, I., Izal, M. y Márquez, M. (2006). Estudio e intervención sobre el malestar psicológico de los cuidadores de personas con demencia: el papel de los pensamientos disfuncionales. Ministerio de Trabajo y Asuntos Sociales: Madrid.

Marco, E. (2004). Calidad de Vida del Cuidador del Hemipléjico Vascular. Tesis doctoral sin publicar, Universidad Autónoma de Barcelona.

Marmaneu-Moliner, (2007, junio). Compartiendo Ideas. En mente, 1, p. 3.

Martín, M., Salvado, S., Mijo L. Rico, J., Lanz, P. y Taussig, M. (1996). Adaptación para el medio español de la Escala de Sobrecarga del Cuidador (Caregiver Burden Interview) de Zarit. Revista Gerontología, 6, 338-346.

Montorio, I., Yanguas J. y Díaz, P., (2000). El cuidado del anciano en el hogar. En Izal, M. y Montorio, I. (eds.). Gerontología Conductual: Bases para la intervención y ámbitos de aplicación. Madrid: Síntesis

Muela, J., Torres, C. y Peláez, E. (2002). Nuevo instrumento de evaluación de situaciones estresantes en cuidadores de enfermos con Alzheimer. Anales de Psicología, 18, 319-331.

Neundorfer, M. (1991). Coping and health outcomes in spouses caregivers of persons with dementia. Nursing Research, 40, 260-265.

Pearlin, L. (1994). Conceptual strategies for the study of caregivers stress. En Light, E. (ed.). Stress effects on family caregivers of Alzheimer's patients. New York. Springer.

Pearlin, L. \& Schooler, C. (1978). The Structure of Coping. Journal of Health and Social Behavior, 22, 337-356.

Pearlin, Mullan, Semple \& Skaff (1990). Caregiving and stress process: an overview of concepts and their measures. Gerontologist, $30(5), 583-59$.

Rodríguez del Álamo, A. (2002). Factores de riesgo de sobrecarga en los familiares cuidadores de enfermos de Alzheimer. Alzheimer, 28 (4), 34-6.

Rodríguez del Álamo, A. (2005). Sobrecarga psicofísica en cuidadores de enfermos de Alzheimer: causas, problemas y soluciones. Fundación SPF de Neurociencias: Barcelona.

Roger, D. (1995). The mechanics of stress: a model for the relationship between stress, health and personality. Comunicación 
presentada en la 5ta Conferencia Internacional en manejo de Estrés, Noordwijkehout, Holanda.

Roger, D., Jarvis, G. \& Najarian, B. (1993). Detachment and coping: the construction and validation of a new scale for measuring coping strategies. Personality and Individual Differences, 15, 619-626.

Roig, M., Abengozar, M. y Serra, E. (1998). La sobrecarga en los cuidadores principales de enfermos de Alzheimer. Anales de Psicología. 14 (2), 215-227.

Santed, M., Sandin, B., Chorot, P., Olmedo, M. y García-Campayo, J. (2003). The role of negative and positive affectivity on perceived stress, subjetive health relationships. Acta Neuropsychiatrica, 15, 199-216.

Velásquez (2006). Área de los aspectos socio-familiares. En Ventura, R. (ed.). 400 respuestas a 400 preguntas sobre la demencia: Una guía para el profesional de la Salud. Bibliomédica: Montevideo. Zarit, S. (1986). Subjetive Burden of husbands and wives as caregivers: a longitudinal study. Gerontologist, 26 (3), 260-266.

Zarit, S. \& Knight, B. (1996). A guide to psychotherapy and aging. Effective clinical interventions in a life-stage context. Washington: APA. 